\title{
AVERAGE OPERATORS ON RECTANGULAR HERZ SPACES
}

\author{
Carolina Espinoza-Villalva - Martha Guzmán-Partida
}

\begin{abstract}
We introduce a family of Herz type spaces considering rectangles instead of balls and we study continuity properties of some average operators acting on them.
\end{abstract}

\section{Introduction}

Herz spaces have been studied for many years. The roots of this subject lie on the pioneering work of $\mathrm{N}$. W i en er [11], A. B e ur ling [2] and C. Herz [9]. Later, these spaces were generalized by other mathematicians in order to study continuity properties of classical operators in harmonic analysis, as well as to develop local versions of Hardy spaces and bounded mean oscillation spaces.

There are several definitions of Herz space. The following is classical and corresponds to the inhomogeneous setting: a measurable function $f$ belongs to the Herz space $K_{p, q}^{\alpha}\left(\mathbb{R}^{n}\right), 1 \leq p, q<\infty, \alpha \in \mathbb{R}$ if

$$
\|f\|_{K_{p, q}^{\alpha}}:=\left(\sum_{k=0}^{\infty} 2^{n k \alpha q}\left\|f \chi_{C_{k}}\right\|_{p}^{q}\right)^{1 / q}<\infty
$$

and for $q=\infty$,

$$
\|f\|_{K_{p, \infty}^{\alpha}}:=\sup _{k \geq 0}\left(2^{n k \alpha}\left\|f \chi_{C_{k}}\right\|_{p}\right)<\infty .
$$

Here, $C_{0}$ is the open unit ball $B_{1}(0)$ and $C_{k}=B_{2^{k}}(0) \backslash B_{2^{k-1}}(0), k \in \mathbb{N}$.

Setting $\alpha=-1 / p$ in (2), we obtain the space $B^{p}\left(\mathbb{R}^{n}\right)$ that also can be characterized by mean of the condition [5], [7]

$$
\sup _{R \geq 1}\left(\frac{1}{\left|B_{R}(0)\right|} \int_{B_{R}(0)}|f(x)|^{p} \mathrm{~d} x\right)^{1 / p}<\infty
$$

(C) 2016 Mathematical Institute, Slovak Academy of Sciences. 2010 Mathematics Subject Classification: 42B35, 26D10.

Keywords: Herz spaces, average operator. 


\section{CAROLINA ESPINOZA-VILLALVA — MARTHA GUZMÁN-PARTIDA}

and the quantity on the left hand side of (3) defines an equivalent norm to $\|f\|_{K_{p, \infty}^{-1 / p}}$ that is usually denoted by $\|f\|_{B^{p}}$. With any of these norms, $B^{p}\left(\mathbb{R}^{n}\right)$ turns out to be a Banach space. Moreover, for $1 \leq p_{1}<p_{2}<\infty$ we have the inclusions $B^{p_{2}}\left(\mathbb{R}^{n}\right) \subset B^{p_{1}}\left(\mathbb{R}^{n}\right)$ and $L^{\infty}\left(\mathbb{R}^{n}\right) \subset B^{p}\left(\mathbb{R}^{n}\right)$ for every $p$.

In this work we will restrict to the context of the space $B^{p}\left(\mathbb{R}^{n}\right)$ for $1 \leq p<\infty$. Our aim is to explore what happens when we consider rectangles with sides parallel to the coordinate axes instead of balls in (3). As we will see below, although we obtain a smaller space than $B^{p}\left(\mathbb{R}^{n}\right)$, it is still appropriate to study continuity properties of some classical operators. In the context of the present paper, we study continuity properties of some discrete and continuous versions of the classical Hardy average operator. This operator has been extensively studied by many authors on different function spaces. We restrict ourself to consider the most simple versions of this operator in order to make the reading of the present paper easy.

The manuscript is organized as follows: the second section is devoted to introduce the rectangular Herz spaces and to give some examples. In the third section we introduce average operators to be considered and we prove the continuity of these averages on our spaces.

We will employ standard notation along this work and we will also adopt the convention to denote a constant that could be changing line by line by $C$.

\section{Rectangular Herz spaces}

For $1 \leq p<\infty$, we define the following space

$$
\mathcal{B}^{p}\left(\mathbb{R}^{n}\right)=\left\{f \in L_{l o c}^{p}\left(\mathbb{R}^{n}\right):\|f\|_{\mathcal{B}^{p}}<\infty\right\},
$$

where

$$
\|f\|_{\mathcal{B}^{p}}:=\sup _{\substack{R_{j} \geq 1 \\ j=1, \ldots, n}}\left[\frac{1}{R_{1} \cdots R_{n}} \int_{\left[-R_{1}, R_{1}\right] \times \cdots \times\left[-R_{n}, R_{n}\right]}|f(x)|^{p} \mathrm{~d} x\right]^{1 / p} .
$$

If the context does not cause confusion, we will simply write $\mathcal{B}^{p}$. Notice that for $n=1$, the spaces $\mathcal{B}^{p}(\mathbb{R})$ and $B^{p}(\mathbb{R})$ coincide.

Standard arguments (see [1], for example) allow us to see that $\left(\mathcal{B}^{p},\|\cdot\|_{\mathcal{B}^{p}}\right)$ is

a Banach space. Moreover, it is clear that $\mathcal{B}^{p} \subset B^{p}$ and $\|\cdot\|_{B^{p}} \leq\|\cdot\|_{\mathcal{B}^{p}}$ since Lebesgue measure of balls and cubes are comparable.

Proposition 1. The space $\mathcal{B}^{p}\left(\mathbb{R}^{n}\right)$ is properly contained in $B^{p}\left(\mathbb{R}^{n}\right)$ when $n \geq 2$. 


\section{AVERAGE OPERATORS ON RECTANGULAR HERZ SPACES}

Pr o of. For the sake of clarity, we will consider the case $n=2$.

Let $f: \mathbb{R}^{2} \rightarrow \mathbb{R}$ be the function defined as follows:

$$
f(x)=\left\{\begin{array}{clll}
0 & \text { if } & x \notin([-1,1] \times \mathbb{R}) \cup(\mathbb{R} \times[-1,1]), \\
1 & \text { if } & x \in([-1,1] \times[-1,1]), \\
2^{1 / p} \quad \text { if } & x & \in([-1,1] \times(1,2]) \\
& & \cup([-1,1] \times[-2,-1)) \\
& & \cup((1,2] \times[-1,1]) \\
& & \cup([-2,-1) \times[-1,1]), \\
& \vdots & & \\
n^{1 / p} \quad \text { if } & x & \in([-1,1] \times(n-1, n]) \\
& & \cup([-1,1] \times[-n,-n+1)) \\
& & \cup((n-1, n] \times[-1,1]) \\
& \cup([-n,-n+1) \times[-1,1]), & n \geq 2 .
\end{array}\right.
$$

Take $R \geq 1$. We can find $k \in \mathbb{N}$ such that $k \leq R<k+1$ and thus

$$
\begin{aligned}
\frac{1}{\left|[-R, R]^{2}\right|} \int_{[-R, R]^{2}}|f(x)|^{p} \mathrm{~d} x & \leq \frac{1}{4 k^{2}} \int_{[-(k+1), k+1]^{2}}|f(x)|^{p} \mathrm{~d} x \\
& =\frac{1}{4 k^{2}}\left[1 \cdot 2^{2}+2 \cdot 2^{3}+3 \cdot 2^{3}+\cdots+(k+1) \cdot 2^{3}\right] \\
& \leq \frac{2}{k^{2}}[1+2+\cdots+(k+1)] \\
& =\frac{(k+1)(k+2)}{k^{2}} \leq 6
\end{aligned}
$$

which shows that $f \in B^{p}\left(\mathbb{R}^{2}\right)$. However, if we now consider rectangles of the form $[-1,1] \times[-L, L]$ for $L \geq 2$, we can pick $m \in \mathbb{N}$ such that $m \leq L<m+1$ and therefore

$$
\begin{aligned}
\frac{1}{|[-1,1] \times[-L, L]|} \int_{[-1,1] \times[-L, L]}|f(x)|^{p} \mathrm{~d} x & =\frac{1}{4 L} \int_{[-1,1] \times[-L, L]}|f(x)|^{p} \mathrm{~d} x \\
& \geq \frac{1}{4(m+1)} \int_{[-1,1] \times[-m, m]}|f(x)|^{p} \mathrm{~d} x \\
& =\frac{1}{4(m+1)}\left[1 \cdot 2^{2}+2 \cdot 2^{2}+\cdots+m \cdot 2^{2}\right] \\
& =m / 2 \rightarrow \infty \quad \text { if } m \rightarrow \infty,
\end{aligned}
$$

that is, $f \notin \mathcal{B}^{p}\left(\mathbb{R}^{2}\right)$. 


\section{CAROLINA ESPINOZA-VILLALVA — MARTHA GUZMÁN-PARTIDA}

Using the idea of the previous example, we can get a characterization of the space $\mathcal{B}^{p}\left(\mathbb{R}^{n}\right)$. To this end, consider the following subsets of $\mathbb{R}^{n}$ :

$$
C_{j_{1}, j_{2}, \ldots, j_{n}}=C_{j_{1}} \times C_{j_{2}} \times \cdots \times C_{j_{n}}
$$

where

$$
C_{0}=[-1,1] \quad \text { and } \quad C_{j}=\left\{x \in \mathbb{R}: 2^{j-1}<|x| \leq 2^{j}\right\} \quad \text { for } \quad j \in \mathbb{N} .
$$

For $1 \leq p<\infty$ and $f \in L_{l o c}^{p}\left(\mathbb{R}^{n}\right)$ define

$$
\|f\|_{\mathcal{B}^{p}}^{*}:=\sup _{\substack{j_{i} \geq 0 \\ i=1,2, \ldots, n}} 2^{-\frac{\left(j_{1}+j_{2}+\cdots+j_{n}\right)}{p}}\left\|f \chi_{C_{j_{1}, j_{2}, \ldots, j_{n}}}\right\|_{p} .
$$

Now, we can state the following characterization.

Proposition 2. $f \in \mathcal{B}^{p}\left(\mathbb{R}^{n}\right)$ if and only if $\|f\|_{\mathcal{B}^{p}}^{*}<\infty$. Moreover, $\|f\|_{\mathcal{B}^{p}}$ and $\|f\|_{\mathcal{B}^{p}}^{*}$ are equivalent norms.

P r o o f. Assume that $\|f\|_{\mathcal{B}^{p}}^{*}<\infty$. For $i=1, \ldots, n$, let $R_{i}>1$ and choose $j_{i} \in \mathbb{N}$ such that

We have that

$$
2^{j_{i}-1}<R_{i} \leq 2^{j_{i}}
$$

$$
\begin{aligned}
\int|f(x)|^{p} \mathrm{~d} x & \leq \sum_{k_{1}=0}^{j_{1}} \sum_{\prod_{2}=0}^{j_{2}} \cdots \sum_{k_{n}=0}^{j_{n}} \int|f(x)|^{p} \mathrm{~d} x \\
& \leq \sum_{k_{k_{1}}, k_{2}, \ldots, k_{n}} \sum_{k_{1}=0}^{j_{1}} \cdots \sum_{k_{2}=0}^{j_{2}} \cdots \sum_{k_{n}=0}^{j_{n}} 2^{k_{1}+k_{2}+\cdots+k_{n}}\left(\|f\|_{\mathcal{B}^{p}}^{*}\right)^{p} \\
& \leq C 2^{j_{1}+j_{2}+\cdots+j_{n}}\left(\|f\|_{\mathcal{B}^{p}}^{*}\right)^{p} \\
& \leq C R_{1} R_{2} \cdots R_{n}\left(\|f\|_{\mathcal{B}^{p}}^{*}\right)^{p} .
\end{aligned}
$$

Hence, $f \in \mathcal{B}^{p}\left(\mathbb{R}^{n}\right)$ and $\|f\|_{\mathcal{B}^{p}} \leq C\|f\|_{\mathcal{B}^{p}}^{*}$.

Conversely, if $f \in \mathcal{B}^{p}\left(\mathbb{R}^{n}\right), i=1, \ldots, n$ and $j_{i} \geq 0$,

$$
\begin{aligned}
\left\|f \chi_{C_{j_{1}, j_{2}, \ldots, j_{n}}}\right\|_{p}^{p} & =\underset{\prod_{i=1}^{n}\left[-2^{\left.j_{i}, 2^{j_{i}}\right]}\right.}{ }|f(x)|^{p} \mathrm{~d} x \\
& \leq C\|f\|_{\mathcal{B}^{p}}^{p} 2^{j_{1}+j_{2}+\cdots+j_{n}}
\end{aligned}
$$

which implies that

$$
\|f\|_{\mathcal{B}^{p}}^{*}=\sup _{\substack{j_{i} \geq 0 \\ i=1,2, \ldots, n}} 2^{-\frac{\left(j_{1}+j_{2}+\cdots+j_{n}\right)}{p}}\left\|f \chi_{C_{j_{1}, j_{2}, \ldots, j_{n}}}\right\|_{p} \leq C\|f\|_{\mathcal{B}^{p}} .
$$

This concludes the proof. 


\section{AVERAGE OPERATORS ON RECTANGULAR HERZ SPACES}

\section{Continuity of average operators}

Average integral operators were considered by $\mathrm{H}$ a r d y, L i t t le w o o d and $\mathrm{P}$ óly a in [8]. They proved the following classical inequality:

$$
\int_{0}^{\infty}\left(\frac{F(x)}{x}\right)^{p} \mathrm{~d} x \leq\left(\frac{p}{p-1}\right)^{p} \int_{0}^{1} f^{p}(x) \mathrm{d} x,
$$

where $1<p<\infty, F(x)=\int_{0}^{x} f(t) \mathrm{d} t, f \geq 0$ and the constant $\left(\frac{p}{p-1}\right)^{p}$ is the best possible.

The operator $H_{\varphi}$ introduced by Carton- Lebrun and Fosset in [3] and by $\mathrm{X}$ i a o in [10] is closely related to this operator, which is pointwisely defined as follows:

$$
H_{\varphi} f(x):=\int_{0}^{1} f(t x) \varphi(t) \mathrm{d} t .
$$

$\mathrm{X}$ i a o in [10] proved continuity of $H_{\varphi}$ under appropriate conditions on $\varphi$ on $L^{p}\left(\mathbb{R}^{n}\right)$ and $B M O\left(\mathbb{R}^{n}\right)$ for $1 \leq p \leq \infty$. It is our goal to prove continuity of this and other related operators in our rectangular Herz spaces.

We will start by considering the following discrete version of (5).

Let $\left\{r_{k}\right\}_{k=1}^{\infty}$ be a sequence in $(0,1]$ which is strictly decreasing and $\lim _{k \rightarrow \infty} r_{k}=0$. If $f: \mathbb{R}^{n} \longrightarrow \mathbb{R}$ is a Lebesgue measurable function and $\varphi:\left\{r_{k}: k \in \mathbb{N}\right\} \longrightarrow(0, \infty)$ is any function, consider the operator $H_{\varphi}^{d}$ formally defined as

$$
H_{\varphi}^{d} f(x)=\sum_{k=1}^{\infty} \varphi\left(r_{k}\right) f\left(r_{k} x\right) .
$$

Now, notice that a necessary and sufficient condition for the existence of $H_{\varphi}^{d}$ as a bounded operator on $L^{p}\left(\mathbb{R}^{n}\right)$ is that

$$
\sum_{k=1}^{\infty} r_{k}^{-n / p} \varphi\left(r_{k}\right)<\infty .
$$

Indeed, assuming the convergence of the series in (6) , given $f \in L^{p}\left(\mathbb{R}^{n}\right)$, $1 \leq p<\infty$, and using Minkowski inequality, we obtain

which implies that

$$
\begin{aligned}
\left\|H_{\varphi}^{d} f\right\|_{p} & \leq \sum_{k=1}^{\infty} \varphi\left(r_{k}\right)\left(\int_{\mathbb{R}^{n}}\left|f\left(r_{k} x\right)\right|^{p} \mathrm{~d} x\right)^{1 / p} \\
& =\|f\|_{p} \sum_{k=1}^{\infty} r_{k}^{-n / p} \varphi\left(r_{k}\right),
\end{aligned}
$$

$$
\left\|H_{\varphi}^{d}\right\|_{L^{p} \rightarrow L^{p}} \leq \sum_{k=1}^{\infty} r_{k}^{-n / p} \varphi\left(r_{k}\right) .
$$




\section{CAROLINA ESPINOZA-VILLALVA — MARTHA GUZMÁN-PARTIDA}

Conversely, if $H_{\varphi}^{d}$ is bounded on $L^{p}\left(\mathbb{R}^{n}\right)$, as $\mathrm{X}$ i a o in [10, we can consider the function

$$
f_{\varepsilon}(x)=|x|^{-\frac{n}{p}-\varepsilon} \chi_{\{|x|>1\}},
$$

where $0<\varepsilon<1$. It turns out that $\left\|f_{\varepsilon}\right\|_{p}=\frac{C_{n}}{p \varepsilon}, C_{n}$ an $n$-dimensional constant and

$$
H_{\varphi}^{d} f_{\varepsilon}(x)=\left(\sum_{k=1}^{\infty} r_{k}^{-\frac{n}{p}-\varepsilon} \varphi\left(r_{k}\right)\right)|x|^{-\frac{n}{p}-\varepsilon} \chi_{\{|x|>1\}} .
$$

Thus, the same procedure as done in [10] shows that

and therefore,

$$
\left\|H_{\varphi}^{d}\right\|_{L^{p} \rightarrow L^{p}}^{p}\left\|f_{\varepsilon}\right\|_{p}^{p} \geq\left[\varepsilon^{\varepsilon} \sum_{k=1}^{\infty} r_{k}^{-\frac{n}{p}-\varepsilon} \varphi\left(r_{k}\right)\right]^{p}\left\|f_{\varepsilon}\right\|_{p}^{p}
$$

$$
\left\|H_{\varphi}^{d}\right\|_{L^{p} \rightarrow L^{p}} \geq\left[\varepsilon^{\varepsilon} \sum_{k=1}^{\infty} r_{k}^{-\frac{n}{p}-\varepsilon} \varphi\left(r_{k}\right)\right] \geq \varepsilon^{\varepsilon} \sum_{k=1}^{\infty} r_{k}^{-\frac{n}{p}} \varphi\left(r_{k}\right)
$$

for any $0<\varepsilon<1$. Now, letting $\varepsilon \rightarrow 0$, we obtain

$$
\left\|H_{\varphi}^{d}\right\|_{L^{p} \rightarrow L^{p}} \geq \sum_{k=1}^{\infty} r_{k}^{-\frac{n}{p}} \varphi\left(r_{k}\right) .
$$

We have proved the following result.

Theorem 3. The operator $H_{\varphi}^{d}$ is a bounded operator on $L^{p}\left(\mathbb{R}^{n}\right), 1 \leq p<\infty$, if and only if $\sum_{k=1}^{\infty} r_{k}^{-\frac{n}{p}} \varphi\left(r_{k}\right)<\infty$. In such a case,

$$
\left\|H_{\varphi}^{d}\right\|_{L^{p} \rightarrow L^{p}}=\sum_{k=1}^{\infty} r_{k}^{-\frac{n}{p}} \varphi\left(r_{k}\right) .
$$

We can also consider the following generalization of the operator $H_{\varphi}^{d}$.

Let $\Phi:\left\{r_{k_{1}}^{(1)}: k_{1} \in \mathbb{N}\right\} \times \cdots \times\left\{r_{k_{n}}^{(n)}: k_{n} \in \mathbb{N}\right\} \longrightarrow(0, \infty)$ be any function where, for every $j=1, \ldots, n$, the sequence $\left\{r_{k_{j}}^{(j)}\right\}_{k_{j}=1}^{\infty} \subset(0,1]$ is strictly decreasing and $\lim _{k_{j} \rightarrow \infty} r_{k_{j}}^{(j)}=0$. For a Lebesgue measurable function $f: \mathbb{R}^{n} \longrightarrow \mathbb{R}$, define formally

$$
\mathbb{H}_{\Phi}^{d} f(x)=\sum_{k_{1}=1}^{\infty} \cdots \sum_{k_{n}=1}^{\infty} \Phi\left(r_{k_{1}}^{(1)}, \ldots, r_{k_{n}}^{(n)}\right) f\left(r_{k_{1}}^{(1)} x_{1}, \ldots, r_{k_{n}}^{(n)} x_{n}\right) .
$$

With the same proof as in Theorem 3, we can show:

TheOrem 4. The operator $\mathbb{H}_{\Phi}^{d}$ is a bounded operator on $L^{p}\left(\mathbb{R}^{n}\right), 1 \leq p<\infty$, if and only if

$$
\sum_{k_{1}=1}^{\infty} \ldots \sum_{k_{n}=1}^{\infty} \Phi\left(r_{k_{1}}^{(1)}, \ldots, r_{k_{n}}^{(n)}\right)\left(r_{k_{1}}^{(1)}\right)^{-1 / p} \ldots\left(r_{k_{n}}^{(n)}\right)^{-1 / p}<\infty .
$$


In such a case,

$$
\left\|\mathbb{H}_{\Phi}^{d}\right\|_{L^{p} \rightarrow L^{p}}=\sum_{k_{1}=1}^{\infty} \cdots \sum_{k_{n}=1}^{\infty} \Phi\left(r_{k_{1}}^{(1)}, \ldots, r_{k_{n}}^{(n)}\right)\left(r_{k_{1}}^{(1)}\right)^{-1 / p} \ldots\left(r_{k_{n}}^{(n)}\right)^{-1 / p}
$$

Now, we will study the action of the operator $\mathbb{H}_{\Phi}^{d}$ on our rectangular Herz spaces defined in the previous section.

For these spaces, the proof of the continuity of the operator $\mathbb{H}_{\Phi}^{d}$ is even easier. We provide it for the sake of completeness.

TheOREM 5. The operator $\mathbb{H}_{\Phi}^{d}$ is a bounded operator on $\mathcal{B}^{p}\left(\mathbb{R}^{n}\right), 1 \leq p<\infty$, if and only if

$$
\sum_{k_{1}=1}^{\infty} \cdots \sum_{k_{n}=1}^{\infty} \Phi\left(r_{k_{1}}^{(1)}, \ldots, r_{k_{n}}^{(n)}\right)<\infty
$$

In such a case,

$$
\left\|\mathbb{H}_{\Phi}^{d}\right\|_{\mathcal{B}^{p} \rightarrow \mathcal{B}^{p}}=\sum_{k_{1}=1}^{\infty} \cdots \sum_{k_{n}=1}^{\infty} \Phi\left(r_{k_{1}}^{(1)}, \ldots, r_{k_{n}}^{(n)}\right) .
$$

P r o o f. Assuming condition (8), taking $R_{j}>1, j=1, \ldots, n$, and using Minkowski inequality, we can see that

$$
\begin{aligned}
& {\left[\frac{1}{R_{1} \cdots R_{n}} \underset{\left[-R_{1}, R_{1}\right] \times \cdots \times\left[-R_{n}, R_{n}\right]}{\left.\int\left|\mathbb{H}_{\Phi}^{d} f(x)\right|^{p} \mathrm{~d} x\right]^{1 / p}}\right.} \\
& \leq \sum_{k_{1}=1}^{\infty} \cdots \sum_{k_{n}=1}^{\infty} \Phi\left(r_{k_{1}}^{(1)}, \ldots, r_{k_{n}}^{(n)}\right)\left[\frac{1}{R_{1} \cdots R_{n}} \int_{\left[-R_{1}, R_{1}\right] \times \cdots \times\left[-R_{n}, R_{n}\right]}\left|f\left(r_{k_{1}}^{(1)} x_{1}, \ldots, r_{k_{n}}^{(n)} x_{n}\right)\right|^{p} \mathrm{~d} x\right]^{1 / p} \\
& \leq \sum_{k_{1}=1}^{\infty} \cdots \sum_{k_{n}=1}^{\infty} \Phi\left(r_{k_{1}}^{(1)}, \ldots, r_{k_{n}}^{(n)}\right)\|f\|_{\mathcal{B}^{p}},
\end{aligned}
$$

and hence,

$$
\left\|\mathbb{H}_{\Phi}^{d}\right\|_{\mathcal{B}^{p}} \leq \sum_{k_{1}=1}^{\infty} \cdots \sum_{k_{n}=1}^{\infty} \Phi\left(r_{k_{1}}^{(1)}, \ldots, r_{k_{n}}^{(n)}\right)
$$

Now, if the operator $\mathbb{H}_{\Phi}^{d}$ is bounded on $\mathcal{B}^{p}\left(\mathbb{R}^{n}\right)$, it is susfficient to consider the function $f_{0} \equiv 1$ because, in such a case, we easily obtain the required reverse inequality.

Our next goal is to generalize the operator given by (7). Before doing this, we will define another class of rectangular spaces closely related to $\mathcal{B}^{p}$. 


\section{CAROLINA ESPINOZA-VILLALVA — MARTHA GUZMÁN-PARTIDA}

Definition 6. For $1 \leq p<\infty$, we define

$$
\mathcal{C} \mathcal{M O}^{p}\left(\mathbb{R}^{n}\right)=\left\{f \in L_{l o c}^{p}\left(\mathbb{R}^{n}\right):\|f\|_{\mathcal{C M O}^{p}}<\infty\right\}
$$

where

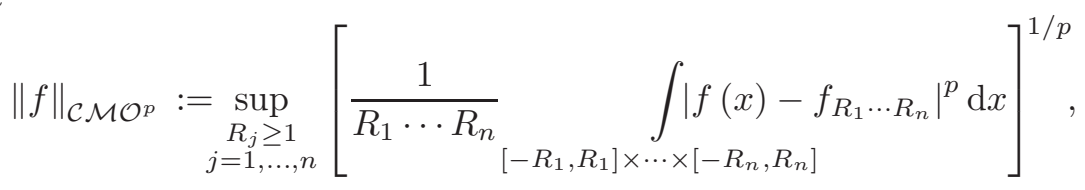

and $f_{R_{1} \ldots R_{n}}$ is the average of $f$ on $\left[-R_{1}, R_{1}\right] \times \cdots \times\left[-R_{n}, R_{n}\right]$.

It is not difficult to show that $\left(\mathcal{C M O}^{p},\|\cdot\|_{\mathcal{C M O}^{p}}\right)$ is a Banach space if we identify functions that differ by a constant almost everywhere on $\mathbb{R}^{n}$. Also, we obtain an equivalent norm to $\|\cdot\|_{\mathcal{C M O}^{p}}$ if we consider the quantity

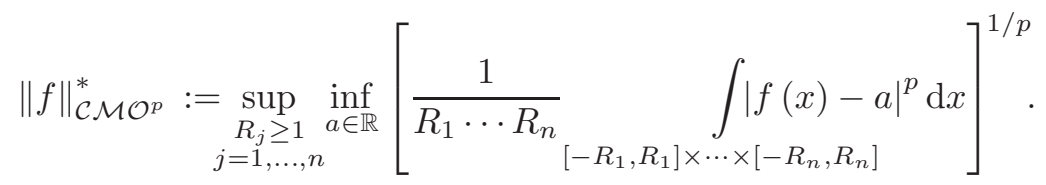

This space is the rectangular version of the space $C M O^{p}$ [4, 7] whose elements satisfy the condition

$$
\sup _{R \geq 1}\left[\frac{1}{|Q(0, R)|} \int_{Q(0, R)}\left|f(x)-f_{Q(0, R)}\right|^{p} \mathrm{~d} x\right]^{1 / p}<\infty .
$$

Here, $Q(0, R)$ denotes the cube centered at 0 with side length equal to $R$. Clearly, $\mathcal{B}^{p} \subset \mathcal{C M O}^{p} \subset C M O^{p}$.

Now, we consider the following operator:

For Lebesgue measurable functions $f: \mathbb{R}^{n} \longrightarrow \mathbb{R}$, and $\phi:[0,1]^{n} \longrightarrow(0, \infty)$, we define

$$
\mathbb{H}_{\phi} f(x):=\int_{[0,1]^{n}} f\left(t_{1} x_{1}, \ldots, t_{n} x_{n}\right) \phi\left(t_{1}, \ldots, t_{n}\right) \mathrm{d} t_{1} \cdots \mathrm{d} t_{n}
$$

Observe that the same proof as given by $\mathrm{Xia}$ o in 10 shows that $\mathbb{H}_{\phi}$ is a bounded operator on $L^{p}\left(\mathbb{R}^{n}\right), 1 \leq p<\infty$, if and only if

$$
\int_{[0,1]^{n}} t_{1}^{-1 / p} \cdots t_{n}^{-1 / p} \phi\left(t_{1}, \ldots, t_{n}\right) \mathrm{d} t_{1} \cdots \mathrm{d} t_{n}<\infty .
$$

We will give equivalent conditions for the boundedness of the operator $\mathbb{H}_{\phi}$ on the spaces $\mathcal{B}^{p}$ and $\mathcal{C} \mathcal{M O}^{p}$. 


\section{AVERAGE OPERATORS ON RECTANGULAR HERZ SPACES}

Theorem 7. The operator $\mathbb{H}_{\phi}$ is a bounded operator on $\mathcal{B}^{p}\left(\mathbb{R}^{n}\right)$ and $\mathcal{C M O}^{p}\left(\mathbb{R}^{n}\right)$, $1 \leq p<\infty$, if and only if

$$
\int_{[0,1]^{n}} \phi\left(t_{1}, \ldots, t_{n}\right) \mathrm{d} t_{1} \cdots \mathrm{d} t_{n}<\infty
$$

Moreover,

$$
\left\|\mathbb{H}_{\phi}\right\|_{\mathcal{B}^{p} \rightarrow \mathcal{B}^{p}}=\left\|\mathbb{H}_{\phi}\right\|_{\mathcal{C M O}^{p} \rightarrow \mathcal{C} \mathcal{M O} \mathcal{O}^{p}}=\int_{[0,1]^{n}} \phi\left(t_{1}, \ldots, t_{n}\right) \mathrm{d} t_{1} \cdots \mathrm{d} t_{n} .
$$

P r o o f. Just for illustration, we prove the equivalence for the space $\mathcal{C} \mathcal{M O}^{p}\left(\mathbb{R}^{n}\right)$.

Suppose that the integral in (11) is finite. Then, for $R_{j}>1, j=1, \ldots, n$ and $f \in \mathcal{C M O}^{p}\left(\mathbb{R}^{n}\right)$, we can easily see that

$$
\left(\mathbb{H}_{\phi} f\right)_{R_{1} \cdots R_{n}}=\int_{[0,1]^{n}} f_{t_{1} R_{1} \cdots t_{n} R_{n}} \phi\left(t_{1}, \ldots, t_{n}\right) \mathrm{d} t_{1} \cdots \mathrm{d} t_{n} .
$$

Now, by Minkowski inequality and an appropriate change of variable, we have that

$$
\begin{aligned}
& {\left[\frac{1}{R_{1} \cdots R_{n}} \underset{\left[-R_{1}, R_{1}\right] \times \cdots \times\left[-R_{n}, R_{n}\right]}{\left.\int\left|\mathbb{H}_{\phi} f(x)-\left(\mathbb{H}_{\phi} f\right)_{R_{1} \cdots R_{n}}\right|^{p} \mathrm{~d} x\right]^{1 / p}}\right.} \\
& \leq \int_{[0,1]^{n}}\left(\frac{1}{R_{1} \cdots R_{n}} \underset{\left[-R_{1}, R_{1}\right] \times \cdots \times\left[-R_{n}, R_{n}\right]}{\int}\left|f\left(t_{1} x_{1}, \ldots, t_{n} x_{n}\right)-f_{t_{1} R_{1} \cdots t_{n} R_{n}}\right|^{p} \mathrm{~d} x\right)^{1 / p} \\
& \quad \times \phi\left(t_{1}, \ldots, t_{n}\right) \mathrm{d} t_{1} \cdots \mathrm{d} t_{n} \\
& \leq\|f\|_{\mathcal{C M O}^{p}} \int_{[0,1]^{n}} \phi\left(t_{1}, \ldots, t_{n}\right) \mathrm{d} t_{1} \cdots \mathrm{d} t_{n},
\end{aligned}
$$

which implies that

$$
\left\|\mathbb{H}_{\phi}\right\|_{\mathcal{C M O}^{p} \rightarrow \mathcal{C M O} \mathcal{O}^{p}} \leq \int_{[0,1]^{n}} \phi\left(t_{1}, \ldots, t_{n}\right) \mathrm{d} t_{1} \cdots \mathrm{d} t_{n} .
$$

For the converse, it suffices to consider the function $f_{0}(x) \equiv 1$.

Finally, it should be remarked that Theorems 5 and 7 remain true if we consider homogeneous versions of the spaces $\mathcal{B}^{p}$ and $\mathcal{C M O}^{p}$, that is, those defined by taking $R_{j}>0$ for every $j=1, \ldots, n$ in (4) and (9). 


\section{CAROLINA ESPINOZA-VILLALVA — MARTHA GUZMÁN-PARTIDA}

\section{REFERENCES}

[1] ALVAREZ, J.-GUZMÁN-PARTIDA, M.-LAKEY, J.: Spaces of bounded $\lambda$-central mean oscillation, Morrey spaces, and $\lambda$-central Carleson measures, Collect. Math. 51 (2000), 1-47.

[2] BEURLING, A.: Construction and analysis of some convolution algebras, Ann. Inst. Fourier (Grenoble) 14 (1964), 1-32.

[3] CARTON-LEBRUN, C.-FOSSET, M.: Moyennes et quotients de Taylor dans BMO, Bull. Soc. Roy. Sci. Liège 53 (1984), 85-87.

[4] CHEN, Y.-LAU, K.: Some new classes of Hardy spaces, J. Funct. Anal. 84 (1989), $255-278$.

[5] FEICHTINGER, H.: An elementary approach to Wiener's third Tauberian theorem on the Euclidean n-space, in: Proc., Conf. at Cortona '84, Sympos. Math., Vol. 29, Academic Press, New York, 1987, pp. 267-301.

[6] FEICHTINGER, H.-WEISZ, F.: Herz spaces and summability of Fourier transforms, Math. Nachr. 281 (2008), 309-324.

[7] GARCÍA-CUERVA, J.: Hardy spaces and Beurling algebras, J. London Math. Soc. 39 (1989), 499-513.

[8] HARDY, G.-LittlewoOd, J. E.-PÓlYA, G.: Inequalities. Cambridge Univ. Press, Cambridge, 1999.

[9] HERZ, C.: Lipschitz spaces and Bernstein's theorem on absolutely convergent Fourier transforms, J. Appl. Math. Mech. 18 (1968), 283-324.

[10] XIAO, J.: $L^{p}$ and BMO bounds of weighted Hardy-Littlewood averages, J. Math. Anal. Appl. 262 (2001), 660-666.

[11] WIENER, N.: Generalized harmonic analysis, Acta Math. 55 (1930), 117-258.

Received January 1, 2015
Departamento de Matemáticas

Universidad de Sonora

Rosales y Luis Encinas

Hermosillo, Sonora, 83000

MÉXICO

E-mail: carolina.espinoza@mat.uson.mx martha@mat.uson.mx 